\title{
Experimental Validation of an Aerodynamic Sensing Scheme for Post-Stall Aerodynamic Moment Characterization
}

\author{
Derrick Yeo ${ }^{1}$, Ella M. Atkins. ${ }^{2}$, Luis P. Bernal ${ }^{3}$ \\ Department of Aerospace Engineering, University of Michigan, Ann Arbor, Michigan, 48109, U.S.A
}

\author{
and \\ Wei Shyy. ${ }^{4}$ \\ Department of Mechanical Engineering, Hong Kong University of Science and Technology, Kowloon, Hong \\ Kong
}

\begin{abstract}
A fixed-wing aircraft that is capable of low airspeed, high angle of attack flight can serve an expanded range of mission goals. A typical propeller-driven small unmanned aircraft can be equipped with an engine that provides greater thrust than airplane weight; in post-stall states, the aircraft then depends on propeller backwash over the tail for control moment generation. Due to this dependence on propeller wash and potentially negligible free-stream airspeed, conventional wind-vector measurements cannot adequately describe the aerodynamic forces and moments acting on the aircraft. This paper describes a distributed pressure sensing system for a small UAS, enabling in-flight estimates of pitch and yaw moments due to the tail surfaces. A wind tunnel test model was developed around an existing flight vehicle with a $1.8 \mathrm{~m}$ wingspan. Embedded pressure measurements were taken across the tail surfaces at low-airspeed high-thrust conditions. Test data is used to develop a method of determining the in-flight aerodynamic pitch and yaw moments due to the tail surfaces on a fixed wing UAS. Through comparisons with torque transducer measurements, the pressure based measurements are shown to provide moment estimates within one standard deviation interval of transducer measurements at hover, thus are capable of providing accurate moment feedback for fixed wing UAS.
\end{abstract}

\begin{tabular}{|c|c|c|c|}
\hline \multicolumn{4}{|c|}{ Nomenclature } \\
\hline$\rho$ & $=$ air density, $\mathrm{kg} / \mathrm{m}^{3}$ & $S$ & $=$ wing area, $\mathrm{m}^{2}$ \\
\hline$b$ & $=$ wingspan & $c$ & $=$ root chord, $\mathrm{m}$ \\
\hline$l_{i}$ & $=$ tail surface panel moment arm, $\mathrm{m}$ & $L$ & $=$ aircraft lift, $\mathrm{N}$ \\
\hline$V_{a c}$ & $=$ aircraft airspeed, $\mathrm{m} / \mathrm{s}$ & $V_{p w}$ & $=$ propeller wash velocity, $\mathrm{m} / \mathrm{s}$ \\
\hline$\alpha$ & $=$ aircraft angle of attack, degree & $\beta$ & $=$ aircraft side slip angle, degrees \\
\hline$M$ & $=$ aircraft pitch moment, $\mathrm{Nm}$ & $C_{M}$ & $=$ aircraft pitch moment \\
\hline$C_{M_{0}}$ & $=$ zero-alpha pitch moment coefficient & $C_{M_{\alpha}}$ & $=$ alpha dependent pitch coefficient \\
\hline$C_{M_{\delta_{e}}}$ & $=$ elevator dependent pitch coefficient & $S_{\text {htail }}$ & $=$ horizontal tail surface area, $\mathrm{m}^{2}$ \\
\hline$C_{M_{a c}}$ & $=$ pitch moment coefficient, wings/fuselage & $N$ & $=$ aircraft yaw moment, $\mathrm{Nm}$ \\
\hline$C_{N}$ & $=$ aircraft yaw moment coefficient & $C_{N_{\delta r}}$ & $=$ rudder dependent yaw moment \\
\hline$C_{N_{a c}}$ & $=$ yaw moment coefficient, wings/fuselage & $C_{N_{\delta_{a}}}$ & $=$ adverse yaw coefficient \\
\hline$C_{N_{\beta}}$ & $=$ sideslip dependent yaw coefficient & $S_{v t a i l}$ & $=$ vertical tail surface area, $\mathrm{m}^{2}$ \\
\hline$\theta_{i}$ & $\begin{array}{l}=i^{\text {th }} \text { panel deflection angle, degrees } \\
=\text { pressure instrumentation calibration factors }\end{array}$ & $\mathrm{P}_{\text {diff }-\mathrm{i}}$ & $=i^{\text {th }}$ pressure measurement \\
\hline
\end{tabular}

\footnotetext{
${ }^{1} \mathrm{PhD}$ student, Aerospace Engineering Dept, University of Michigan, Ann Arbor, MI, AIAA Student Member

${ }^{2}$ Associate Professor, Aerospace Engineering Dept, University of Michigan, Ann Arbor, MI, Associate Fellow

${ }^{3}$ Associate Professor, Aerospace Engineering Dept, University of Michigan, Ann Arbor, MI, AIAA Member

${ }^{4}$ Provost \& Chair Professor, Department of Mechanical Engineering, Hong Kong University of Science and Technology,

also; Adjunct Professor, Department of Aerospace Engineering, University of Michigan, AIAA Fellow

American Institute of Aeronautics and Astronautics
} 


\section{Introduction}

In the last decade, unmanned aircraft systems (UAS) ranging from large to small sizes have been deployed for $\mathbf{I}_{\text {platform/payload validation, science, and surveillance roles traditionally associated with conventional fixed or }}$ rotary wing aircraft [1]. Small aerobatic UAS carrying modest payloads are typically over-powered to the extent that even a single propeller-driven engine is capable of generating a thrust greater than total vehicle weight. This characteristic enables the small UAS to combine the advantages of a fixed wing platform with those of a rotary wing platform, specifically flying with the efficiency of a fixed-wing aircraft while also being capable of hovering over a site of interest. Such a platform can also land or perch without the need for a full landing strip. A small UAS with the ability to operate at high angles of attack and hover as well as perching can have applications across military and commercial sectors. Such a platform could move quickly and efficiently to new locations but still provide stationary close-range reconnaissance when required [2].

This paper presents recent progress in developing an aerodynamic sensing system for an aerobatic fixed wing platform operating outside conventional fixed wing flight envelopes. It describes a series of simulated hover tests in a wind tunnel to examine the ability of an embedded pressure sensor suite to provide real-time control surface aerodynamic moment estimates. Such measurements are critical when the aircraft wind vector is insufficient to produce significant flow across the tail surfaces, but where backwash from the propeller is sufficient to enable continued control by the elevator and rudder. A brief overview of related work is given and the proposed concept is described. The experimental setup and process are then presented followed by an analysis of test results and a summary of key findings.

\section{Background}

Low airspeed, high angle of attack aerodynamics challenges the current paradigm of small UAS instrumentation which integrates inertial measurements supplemented by airspeed as a minimum or more capably a five-hole probe providing air-data measurements that include airspeed, angle of attack, and sideslip. While such systems have been successful in applications involving conventional fixed wing flight within the traditional flight envelope [3,4,5], flight near or beyond the point of stall are subject to large velocity differences in the flow field and flow separation that results in aerodynamics that are unsteady, nonlinear, and sensitive to small changes in flight conditions. These conditions cannot be adequately handled by inertial instrumentation data fed into traditional controllers [6,7] presuming linear relationships between airspeed, angle of attack, and aerodynamic forces/moments. Despite traditional instrumentation limitations, a number of fixed wing UAS have been successfully guided between cruise and hover in the last decade. Green and Oh developed indoor hobby aircraft that could autonomously transition from cruise to hover using inertial measurements and a linearized controller [8] by using airframe properties such as low rotational inertia, high thrust/weight ratios and a control law that ignored wing stall. A similar approach was employed by Frank et al [9] who achieved successful autonomous transitions to hover and docking in a VICON motion capture environment. Johnson et al [10] developed an adaptive controller that enabled autonomous transitions to and from hover. The guidance law used during the transition was similar in formulation to those used in previous work: the commanded inertial pitch angle was set to vertical to achieve the transition. A slow ramp approach and a faster step-change transition were tested and it was noted that both resulted in significant altitude error during the transition. Johnson et al suggested that an airspeed-bleed strategy was a potential solution. In our previous work [11], the use of pressure based aerodynamic sensing to support such a transition guidance phase was proposed. Processing pressure data over an instrumented wing chord, the autopilot was able to detect stall and could reliably bleed airspeed up to the point it was detected before switching control modes. Flight tests [12] verified that expanded aerodynamic data could indeed detect stall directly signaling the need to employ a control law applicable under stall conditions

The concept of pressure-based estimation of the flow field above an airfoil has been a cornerstone of wind tunnel testing, but traditionally most instrumentation has been housed external to the vehicle. Although results in this paper are from wind tunnel testing, the complete pressure sensing package can also be flown as tested. The most related work has therefore focused on enabling closed loop feedback of "onboard" active flow control schemes to alleviate flow separation or emulate control surfaces through the use of flow actuation. One successful example was

implemented by Patel and Corke $[13,14]$ who considered the time domain response from a high bandwidth pressure 
sensor to predict incipient flow separation at the wing leading-edge then trigger the activation of a plasma flow actuator. Under attached flow conditions, Cox et al [15] used pressure based estimates of the lift curve above an airfoil as feedback for an automated cruise flap. NASA has supported a wind tunnel-based implementation of a distributed actuation and sensing array for use on a blended wing body UAS, using a series of pressure measurements to study the effectiveness of a morphing wing control strategy. More information on these tests can be found in [16] and [17]. The AFOSR AVOCET project [18] aims to continuously tailor the pressure distribution and resulting forces and moments across the wing using advanced micro-tuft sensors and hybrid fluidic flow actuators.

The aerodynamic feedback system described in this paper bridges the two efforts described above. The existing active flow control framework developed by Patel et al $[13,14]$ alleviates retreating blade stall in rotorcraft thus expanding the performance envelope. With the AVOCET project, flow measurement and actuation across the wing surfaces are closely coupled to vehicle control and the system aims to achieve a careful tailoring of vorticity distributions in real time to also alleviate gusts and flow disturbances. While the intended purpose of the aerodynamic data presented in this work is to offer additional data for feedback control, our objectives are not to directly affect the flow structure but to provide improvements on how the conventional surfaces can be used.

The experimental approach presented in this paper utilizes a full-scale UAS platform in the University of Michigan's 5'x7' wind tunnel test section. Wind tunnel tests conducted previously on full-scale small UAS have characterized aerodynamic properties [19] then used in novel control strategies [20,21]. Using the actual flight vehicle as a test model allows for testing when the propulsion system is active. Landman et al investigated the longitudinal and lateral aerodynamic characteristics of a small UAS with and without power applied to the propeller [2222]. Recent work by Ol et al tested an aerobatic RC airframe in the presence of prop-wash using force and moment transducer instrumentation and found that high thrust settings at low advance ratios serve to linearize control-surface response [23]. Their results are also supported by this work, with the distinction of this work in the use of onboard instrumentation capable of providing real-time aerodynamic moment feedback in hover and mixed flow (free stream plus prop-wash) conditions.

\section{Experimental Approach}

\section{III.a Aerodynamic Sensing}

The aerodynamic sensing package proposed in this work is based on a set of pressure measurements taken across the aircraft through pressure ports and through multi-hole probes. An overview of the concept is shown in Figure 1. At each indicated location, a pair of pressure ports on the top and bottom surface is connected to a locally-mounted differential pressure sensor. By comparing top versus bottom pressure at the same surface station, local lift estimates can be calculated for each measured region then integrated with other pressure measurements to estimate lift over the entire surface area. Wing-mounted pressure sensors have been previously flight tested with results described in our earlier work [12]. This paper focuses on characterizing the moments associated with prop-wash flow over the instrumented tail section through pressure sensing, and verifying the pressure-based measurements with independent force-torque (FT) transducer measurements.

\section{III.b Wind Tunnel Test Bed}

A dedicated test platform was developed from the Hangar-9 Funtana X100 commercial-off-the-shelf (COTS) radio control (RC) aircraft. In addition to being fully aerobatic, the Funtana also supports an appreciable avionics payload while maintaining sufficient thrust to stably hover, specifically a thrust to weight ratio larger than 1.5 when fully loaded with an avionics payload of 660 grams. The wind tunnel testbed retains all the systems necessary for $\mathrm{RC}$ flight and includes additional internal structure for mounting a six degree-of-freedom force-torque transducer system. The test setup is configured to be mounted in the University of Michigan 5'x7' wind tunnel. An overview of the platform is shown in Figure 2. The key requirements of the Funtana test platform are a propulsion system with thrust exceeding platform weight, a force-torque sensor, embedded aerodynamic sensing system, and onboard data acquisition system capable of supporting wind tunnel and flight tests. For these tests, the primary sensors are the force-torque transducer and instrumented tail section, which are described in more detail below. 


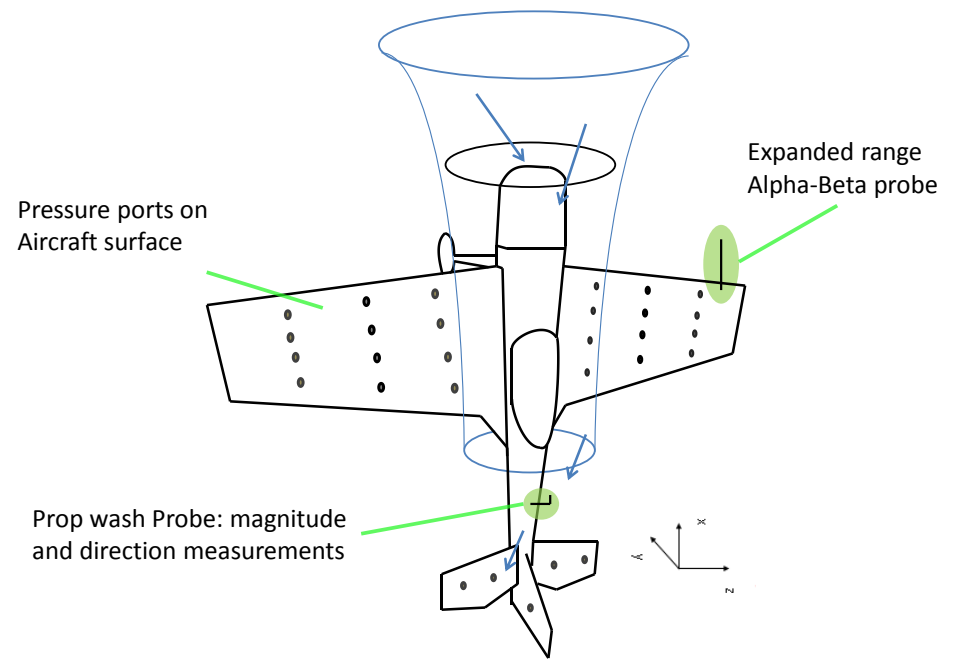

Figure 1: Pressure-Based Aerodynamic Instrumentation Concept

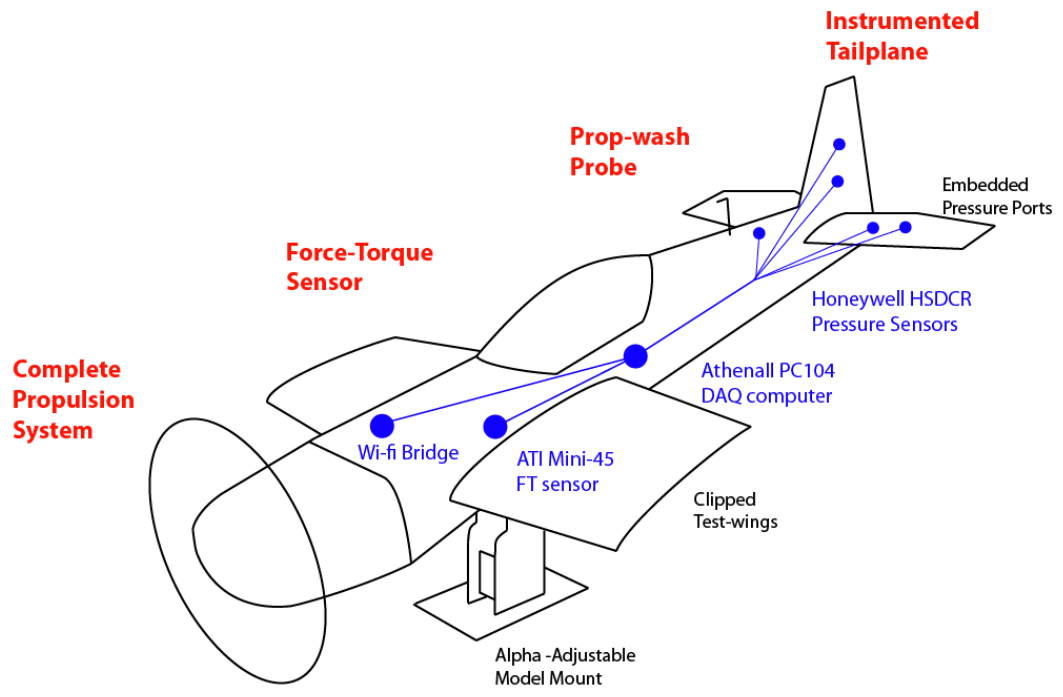

Figure 2: Wind tunnel test platform overview

A Diamond Systems (www.diamondsystems.com) Athena II PC104 computer is used for 16-bit analog data acquisition at $1000 \mathrm{~Hz}(1 \mathrm{kHz})$. The Athena is configured to communicate with an external laptop for DAQ management through a wireless access point. The embedded ATI Industrial Automation (www.ati-ia.com) Mini-45 force-torque sensor has a maximum load capacity of $145 \mathrm{~N}$ in the $x$ (forward) and $y$ directions and $290 \mathrm{~N}$ in the $z$ (vertical) direction. It is mounted internally with the tool-tip facing outwards. This allows the test model to be fully self-contained with no external wiring. The model is mounted on a custom-built, two-part stand that is adjustable for angle-of-attack and features a quick release bolt system to allow the model to be quickly repositioned.

\section{III.c Instrumented Tail Plane}

The tail surfaces on the wind tunnel model are instrumented with pressure sensors embedded within the structure. These provide real time measurements of the differential pressure across the tail surfaces. The pressure ports are distributed across the horizontal stabilizer, elevator, vertical stabilizer and rudder. Each surface is discretized as shown in Figure 3. Lift in each region or grid sector is computed based on the differential pressure 
measurement in that region times the area of that region; total lift of each surface is computed as the sum of lift over all locally-characterized grid regions.

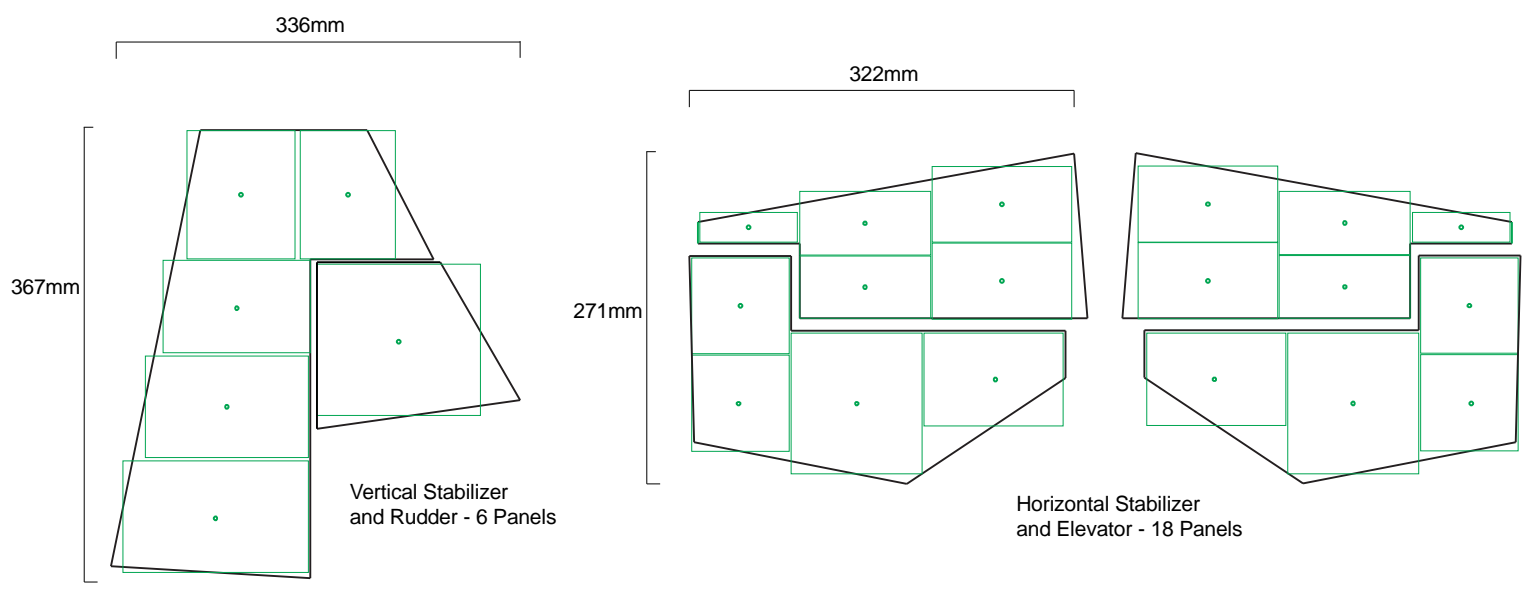

Figure 3: Distributed sensing over tail surface and area discretization scheme

The pressure ports are connected to Honeywell HSCDR 1NDAA5 pressure sensors with a $\pm 1 \mathrm{InH}_{2} \mathrm{O}$ measurement range. These are the most sensitive sensors in the HSCDR series, with the HSCDR series chosen as it is sufficiently small to be located within the surfaces. This allows for short and uniform pressure line lengths between ports and sensors and reduces the low-pass filtering effect identified in our previous work on embedded pressure sensing for a flapping wing platform [24]. To accommodate the instrumentation in the COTS Funtana airframe, additional structure was fabricated and built into the tail surfaces. The horizontal stabilizer and elevators are shown in Figure 4.

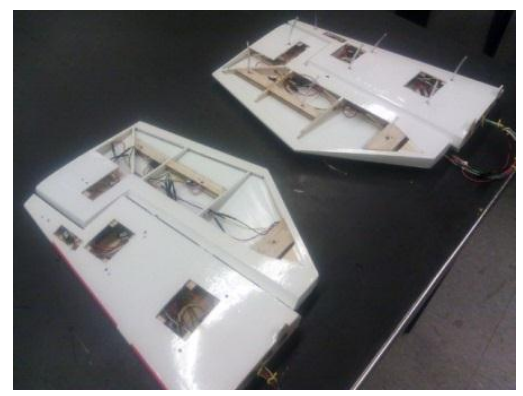

Figure 4: Embedded pressure ports and sensors in the horizontal tail surfaces

\section{III.d Data acquisition and processing}

The wind tunnel experiments enable a comparison between aerodynamic pressure and ATI force-torque (FT) measurements of tail section control moments under controlled and repeatable flow conditions. Flow conditions across the tail surfaces were changed by varying wind tunnel free stream velocity, propeller revolutions per minute (RPM) and airframe mounting angle in the wind tunnel. A series of tail control surface deflection sweeps were carried out over a range of free stream and motor RPM (prop wash) flow conditions. These include an elevator sweep with the rudder at zero deflection, a rudder sweep with the elevator at zero deflection, and a coarse combined rudder/elevator deflection sweep. The deflection ranges and step size were varied for each test condition depending on the maximum moments generated by the control surfaces to accommodate saturation limits of the embedded ATI 
force-torque sensor. A 10 second sampling period was used at each of data point. The force-torque sensor data was time averaged across the sample period and statistics were computed assuming a normal distribution.

Due to the turbulent nature of the flow behind a propeller, the raw pressure data is subject to significant noise that manifests itself as fluctuations about the average value that is representative of a given test condition. The pressure data required additional processing to enable a filter design that can be tailored to provide good performance over the range of expected operational conditions. To allow a more relevant comparison of pressure and transducer based instrumentation, the filter must reduce or eliminate high-frequency fluctuations associated with the propeller while maintaining a high data update rate with minimal data lag. For operations within the wake of a propeller, the most important disturbance time scale will depend on propeller rotation rate as the wake from each propeller blade is convected past the tail surfaces. A simple moving average (SMA) filter that averages data over the passage of two complete propeller revolutions was implemented. It represents the most responsive filter setting that provides for consistent performance over the test range while being sufficiently responsive over all operational conditions.

\section{Pressure Based Moment Estimation}

In the current configuration, distributed sensing across the tail section allows pressures to be measured across the stabilizer and control surfaces. This allows the actual aerodynamic moments induced by the tail to be measured in flight. By taking measurements instead of relying on models, information about a complex flow environment can be more accurately gathered for the future purpose of flight control. A reformulation of the steady flight equations is proposed as part of the overall sensing strategy. The steady flight equations represent the most basic principles of flight mechanics [25] and are used as the foundation for the development of small UAS autopilots. The aerodynamic pitch moment equation for an aircraft in steady flight is

$$
M=\frac{1}{2} \rho V_{a c}{ }^{2} S c C_{M}
$$

By convention, $c$ is the root chord of the wing and $S$ is the surface area of the wing. $\rho$ is atmospheric air density and $V_{a c}$ is the free-stream airspeed of the aircraft. The non-dimensional pitch moment coefficient $C_{m}$ combines the effects of all aerodynamic surfaces such as the wings, fuselage and horizontal stabilizer. It is a linear function of aircraft angle of attack $\alpha$ and elevator deflection $\delta_{e}$ as given in

$$
C_{M}=C_{M_{0}}+C_{M_{\alpha}} \alpha+C_{M_{\delta_{e}}} \delta_{e}
$$

The coefficients $C_{M_{0}}, C_{M_{\alpha}}$, and $C_{M_{\delta_{e}}}$ are determined through theoretical models or wind tunnel testing. Under steady flight conditions where neither the aircraft nor the horizontal tail is stalled, this linear relationship with free stream flow angle is sufficient for the aerodynamic pitch moments to be closely approximated. Similarly, the equation for yaw moment $N$ is

$$
N=\frac{1}{2} \rho V_{a c}^{2} S b C_{N}
$$

where $b$ is the wing span. The yaw moment coefficient $C_{N}$ is a linear function of the aircraft side slip angle $\beta$, aileron deflection $\delta_{a}$ and rudder deflection, $\delta_{r}$ as given in

$$
C_{N_{\text {combined }}}=C_{N_{\beta}} \beta+C_{N_{\delta_{a}}} \delta_{a}+C_{N_{\delta_{r}}} \delta_{r}
$$

where $C_{N_{\delta_{a}}}$ is the yaw moment due to the differential drag caused by aileron deflection. Under the direct measurement scheme, the differential pressure $P_{d i f f-i}$ is measured across the horizontal tail, elevators, vertical tail and rudder at each location ( $i$ ). Each location corresponds to a panel of area $S_{\text {htail }}$ that is $l_{i}$ from the center of gravity and has a deflection angle of $\theta_{i}$. This deflection angle is approximately zero for panels on the stabilizers, and equal to the control surface deflection angle for panels on the rudder and elevator. By considering the moment contributions due to the fuselage and wing combination $C_{M_{a c}}$ and $C_{N_{a c}}$, the terms relating to the tail surfaces can be considered separately as in the following equations 


$$
\begin{gathered}
M=\frac{1}{2} \rho V_{a c}{ }^{2} S b C_{M_{a c}}+A_{M} \sum_{i=1}^{\mathrm{n}_{\text {htail }}} \cos \theta_{i} \cdot \mathrm{P}_{\mathrm{diff}-\mathrm{i}} \cdot S_{\text {htail }_{i}} \cdot l_{i} \\
C_{M_{a c}}=C_{M_{a c_{0}}}+C_{M_{a c_{\alpha}}} \alpha \\
N=\frac{1}{2} \rho V_{a c}{ }^{2} S c C_{N_{a c}}+A_{N} \sum_{i=1}^{\mathrm{n}_{v t a i l}} \cos \theta_{i} \cdot \mathrm{P}_{\mathrm{diff}-\mathrm{i}} \cdot S_{v t a i l_{i}} \cdot l_{i} \\
C_{N_{a c}}=C_{N_{a c_{0}}}+C_{N_{a c_{\beta}}} \beta
\end{gathered}
$$

where the coefficients $A_{M}$ and $A_{N}$ are calibration factors that improve pressure-based estimation of pitch and yaw moments. They allow for systematic errors such as those that may be caused by the coarse discretization scheme. Through direct measurements of aerodynamic moments and knowledge of control surface deflection, control authority can always be determined under uncertain conditions instead of relying on models and offline calibrations. This represents the ability to 'close the loop' with regard to the aerodynamics in complex flow conditions in a manner not possible with air data systems designed to measure free stream flow.

\section{IV.a Calibration of pressure data integration using a low turbulence reference case}

A calibration methodology using a low turbulence free-stream test case was used. By comparing the offsets in slope, a calibration for the aerodynamic instrumentation is computed. This factor was then applied to the pressurebased measurements across all test cases and the corrected results were compared to FT measurements. A low turbulence test case can provide a uniform flow field without the predominant swirl and unsteady effects of the propeller wash. A reference test case was run by using the free stream generated by the wind tunnel with the propeller fixed (zero thrust). The chosen airspeed of approximately $12 \mathrm{~m} / \mathrm{s}$ represents a low-speed cruise condition for the Funtana.

Basic rudder and elevator sweeps were completed and measurements from both sets of instrumentation were compared under low turbulence flow conditions. No additional filtering was performed for the pressure measurements since the propeller was not rotating. These results are shown in Figure 5; note that the FT data for pitching moment was truncated once the sensor saturates between 15-20 degrees elevator deflection. An offset with the control surfaces centered can also be observed. This is most likely due to the moments contributed by the aircraft fuselage and wing and can be described by coefficients $C_{M_{a c}}$ and $C_{N_{a c}}$ in the reformulated equations. Further testing to quantify these coefficients to form a complete aerodynamic model is beyond the scope of this paper.

Using these results as a training case, the coefficients $A_{M}$ and $A_{N}$ were computed by comparing the linear slopes of the pressure-integrated and FT sensor measurements. The linear slopes for each axis were computed by first choosing two control surface deflection angles, $\theta_{1}$ and $\theta_{2}$ on either side of a neutral setting that correspond to moments that are within the linear range of the data. Each $\theta_{i}$ has two corresponding moment measurements from the aerodynamic sensing system and the FT sensor, denoted as $M_{\text {oro }, i}$ and $M_{\text {om }} m_{F T, i}$ respectively. The computation is given in equations (7) to (9) and estimates of the linear slopes and the coefficients for pitch and yaw are summarized in Table 1. 

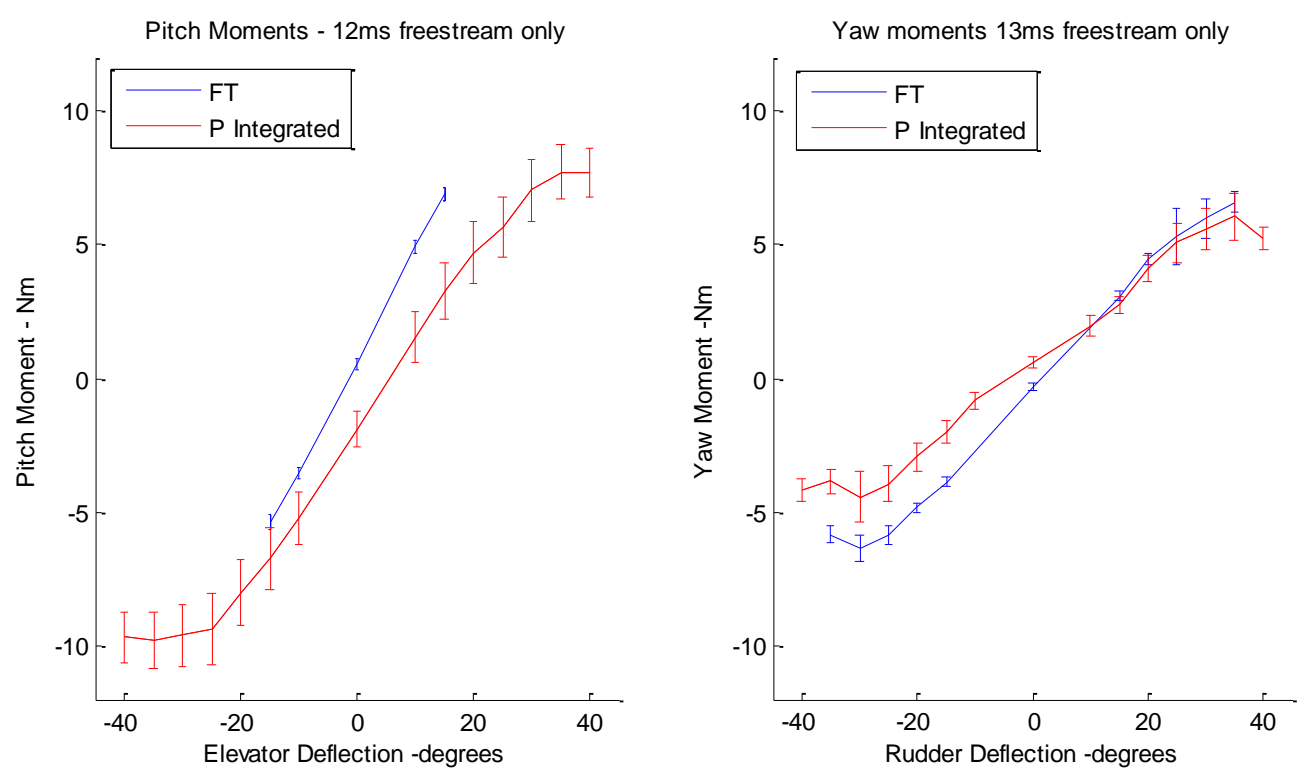

Figure 5: Low turbulence free stream training data set

$$
\begin{gathered}
\text { Slope }_{\text {Aero }_{M, N}}=\frac{\text { Mom }_{\text {Aero }, 2_{M, N}}-\text { Mom }_{\text {Aero }, 1_{M, N}}}{\theta_{2_{M, N}}-\theta_{1_{M, N}}} \\
\operatorname{Slope~}_{F T_{M, N}}=\frac{\operatorname{Mom}_{F T, 2}{ }_{M, N}-\text { Mom }_{F T, 1}{ }_{M, N}}{\theta_{2_{M, N}}-\theta_{1_{M, N}}} \\
A_{M, N}=\frac{\operatorname{Slope}_{F T_{M, N}}}{\text { Slope }_{\text {Aero }_{M, N}}}
\end{gathered}
$$

Table 1: Estimated linear slopes from low-turbulence training case

\begin{tabular}{|c|c|r|c|}
\hline Axis & Slope $_{\text {Aero }_{M, N}}$ & Slope $_{F T} M, N$ & \multicolumn{1}{c|}{$A_{M, N}$} \\
\hline Pitch, $M$ & 19.589 & 24.549 & 1.253 \\
Yaw, $N$ & 9.183 & 13.459 & 1.466 \\
\hline
\end{tabular}

The computed coefficients determined from this 'training set' were used to scale the moments reported by the aerodynamic sensing system in the test cases. If the corrected aerodynamic moment measurements agree well with the FT measurements in the other cases, the same calibration is valid throughout the range of test conditions. 


\section{Propeller Driven Test Results}

The objective of Funtana wind tunnel testing is to extend the ability of onboard aerodynamic sensing to provide real-time estimates of pitch and yaw moments based on flow over the tail. This section presents results from a set of hover tests as well as results from low-airspeed and powered-cruise cases. Test data is summarized in a series of plots that describe the moments measured using both pressure and force-torque instrumentation with changing control-surface deflections.

\section{V.a Hover}

As shown in Figure 6 and Figure 7, the pressure-based moment measurements show good agreement with the FT measurements with overlapping standard deviation intervals at all data points. Calibrated pitch moments at hover are plotted in Figure 6 followed by calibrated yaw moments at hover in Figure 7. Based on these results, it can be concluded that the calibration factors computed in the low-turbulence test case apply to steady hover flight conditions. From the following comparisons, the distributed sensing scheme is shown to accurately measure both pitch and yaw moments when in a steady hover.
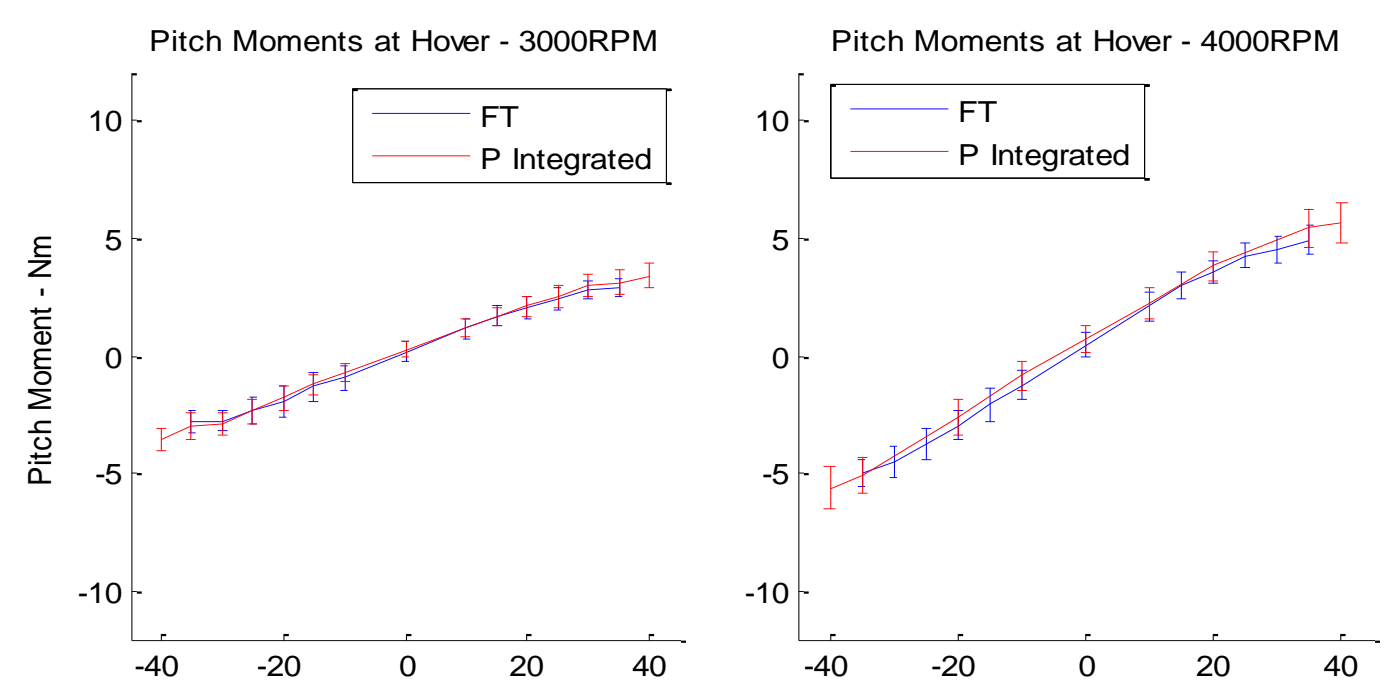

Pitch Moments at Hover - 5000RPM

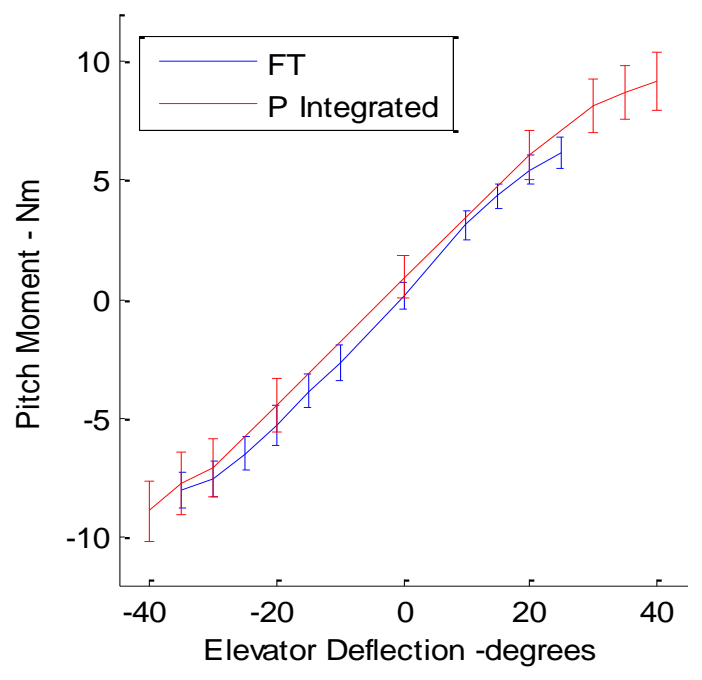

Pitch Moments at Hover - 6000RPM

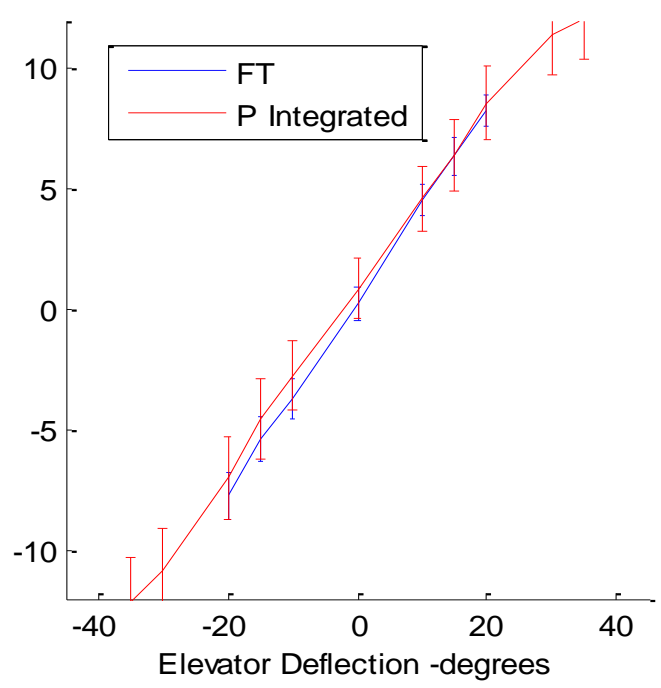

Figure 6: Comparison between corrected pressure-based and FT measurements of hover pitch moments 

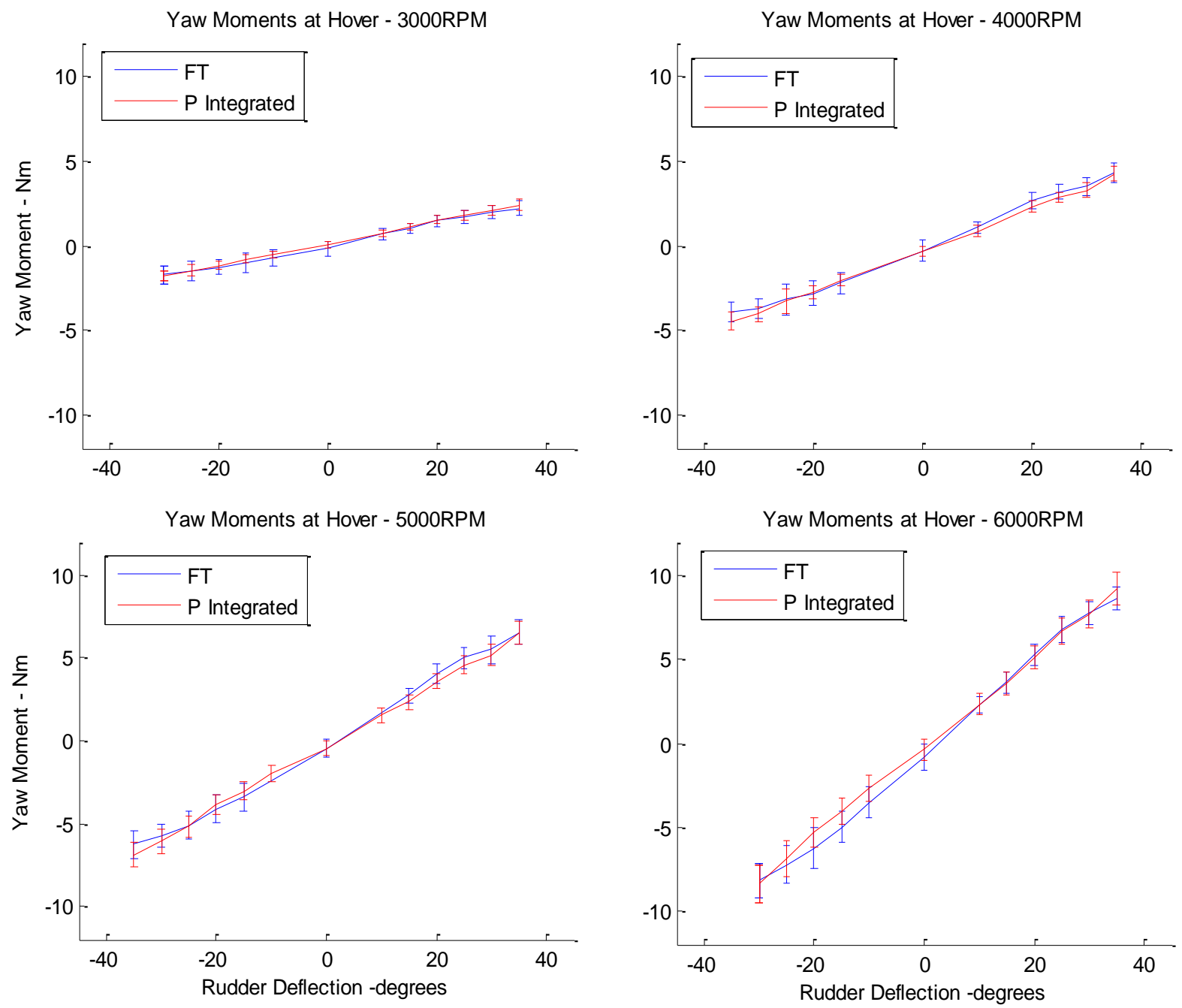

Figure 7: Comparison between corrected pressure-based and FT yaw moment measurements for hover

\section{V.b Alpha25 and 5kCruise Cases}

The calibration was next applied to data from the Alpha25 and 5kCruise cases. In the Alpha25 case, the model is mounted at a 25 degree angle with respect to the test section and the door is shut. The propeller is driven at 5000RPM resulting in a small but measurable free stream through the test section. As an added complication, the tail surfaces are placed near the floor of the test section and are subject to wall interactions. The $5 k C r u i s e$ test case combines an incident free stream at zero angle of attack and sideslip with propeller backwash. This data set allows the sensing system to be evaluated under forward flight conditions. These then compared to FT measurements. The corrected slopes of the pressure based measurements for pitch and yaw are observed to accurately match FT data slopes, as shown in Figure 8 below. While the data point locations agree to within a standard deviation, a distinct offset is also apparent. 

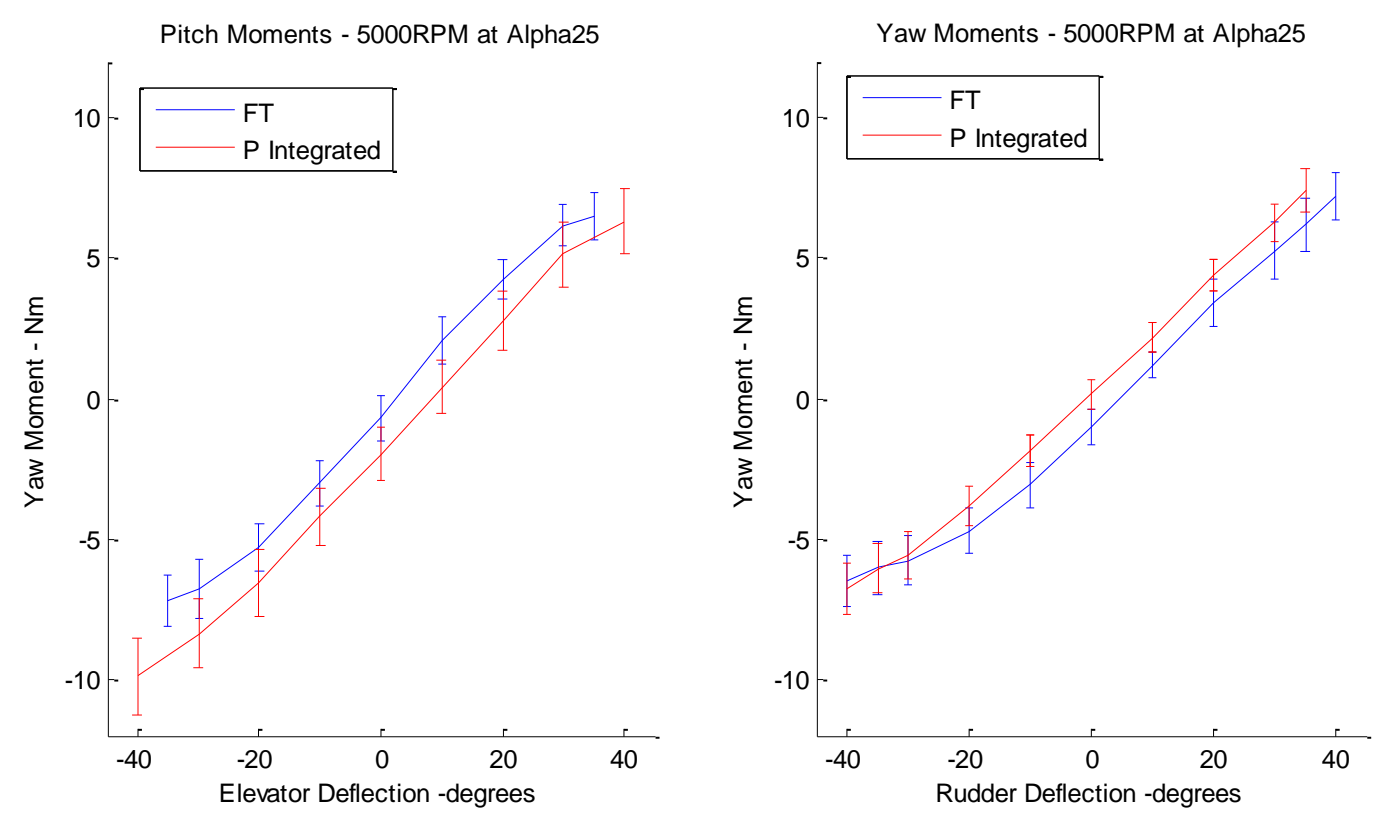

Figure 8: Pressure based measurements at near-hover, Alpha25 case

This offset is most likely due to moment contributions from the wing given the large angle of attack and nonzero free-stream. This offset can be characterized through either a direct sensing scheme or through an experimentally-derived offset coefficient model. The need for such an offset is also apparent in the powered cruise case with lower angle of attack, as shown in Figure 9. Pressure and FT sensor measurements show agreement in slope but with a distinct offset due to contributions from the wing and fuselage. Note that in all tests FT data is truncated where saturation was present.
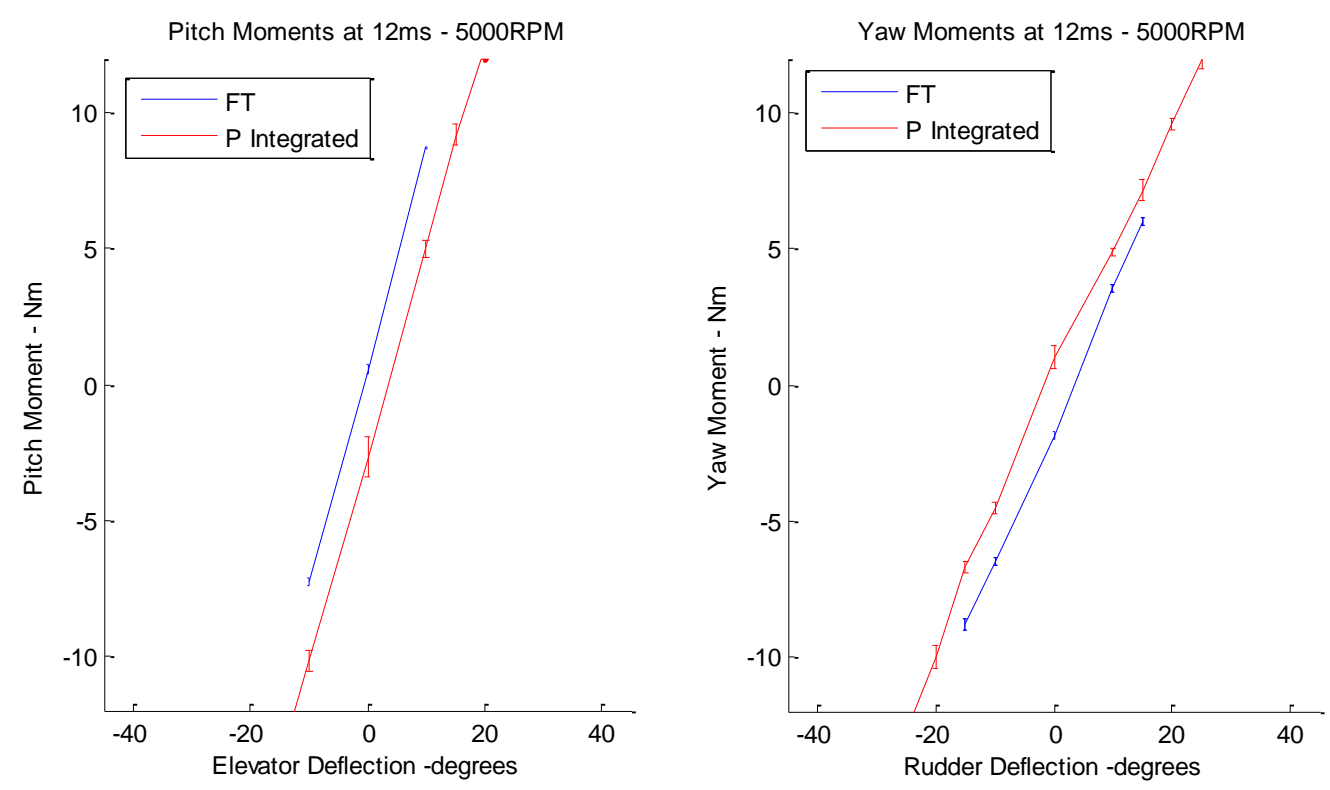

Figure 9: Pressure-based measurements at powered cruise 
The powered cruise test case also demonstrates the ability of the pressure based sensing scheme to account for varying flow regimes. The control authority available in a forward flight situation is far greater than those induced by propeller flow alone as can be noted through much steeper slopes relating control surface deflection and resulting moments. Despite the significant change in flow conditions, the embedded pressure system provides results that are also valid in forward flight. Based on these results, the calibration factors formulated at a low turbulence cruise training data set is observed to be valid at all other test cases.

\section{Conclusions and Future Work}

A direct pressure measurement approach to aerodynamic moment sensing has been proposed and experimentally evaluated for estimating moments induced by the aircraft tail in free stream and hover conditions with propeller prop wash. A reformulation of the steady flight equations allows the integration of distributed sensing across the tail surfaces to provide in-flight estimates of aerodynamic pitch and yaw moments. A calibration scheme is proposed and implemented, showing that a single calibration case with only free stream flow is capable of providing parameter estimates sufficient to also predict pitch and yaw moments from pressure data across a variety of motor RPM and free stream conditions from hover to high angle of attack. While the test results from this paper were obtained in controlled wind tunnel conditions, the direct moment measurement scheme will also enable accurate moment characterization in flight where flow conditions will be less certain. This scheme therefore provides the ability to 'close the loop' with regard to the pitch and yaw moment aerodynamics in complex flow fields in a manner not possible with traditional pitot systems.

As described above, discrepancies in the measured moments due to contributions beyond flow over the tail still exist. In ongoing work, a full set of Funtana airframe stability coefficients is being experimentally determined. A special-purpose probe to measure propeller backwash is also being investigated as a means of providing moment estimates in low-airspeed, high thrust conditions. Further wind tunnel testing across the flight envelope will provide a more complete 'full envelope' model for the Funtana small UAS platform and allow for further development of inflight sensing schemes.

\section{Acknowledgements}

This work has been supported in part by the Boeing Company.

\section{References}

1 Unmanned Aircraft Systems Roadmap 2005-2030, Office of the Secretary of Defense, 2005

2 E. Johnson, A. Wu, J. Neidhoefer, S.Kanna and M. Turbe. "Flight-Test Results of Autonomous Airplane Transitions Between Steady-Level and Hovering Flight," AIAA Journal of Guidance, Control, and Dynamics, Vol. 31, No. 2, March-April 2008

3 S. Herwitz, K. Allmendinger, R. Slye, S. Dunagan, B. Lobitz, L. Johnson, and J. Brass, "Nighttime UAV Vineyard Mission: Challenges of See-and-Avoid in the NAS," Proc. AIAA $3^{\text {rd }}$ Unmanned... Unlimited Conference, Workshop and Exhibit, 20-23 September 2004 Chicago Illinois(AIAA 2004-6417)

4 R. Beard, D. Kingston, M. Quigley, D. Snyder, R. Christiansen, W. Johnson, T. McLain and M. Goodrich, "Autonomous Vehicle Technologies for Small Fixed Wing UAVs," AIAA Journal of Aerospace Computing, Information, and Communication, Vol. 2, January 2005 pp. 92 - 108.

5 R. Hirokawa, D. Kubo, S. Suzuki, J. Meguro and T. Suzuki, "A Small UAV for Immediate Hazard Map Generation," Proc. AIAA Infotech@Aerospace Conference and Exhibit, 7-10 May 2007, Rohnert Park California. (AIAA 2007-2725) 
6 J. Kaloust "Enhanced Stall and Recovery Control System (ESARCS) : A Nonlinear Model-Based Flight Control System For Uninhabited Airborne Vehicles (UAV)," Proc. AIAA $1^{\text {st }}$ Technical Conference and Workshop on Unmanned Aerospace Vehicles, 20-23 May 2002, Portsmouth, Virginia, (AIAA 2002-3489).

7 M. Shields and K. Mohseni, "Limitations of Using the Linearized Equations of Motion for MAV Control," Proc. AIAA Guidance Navigation and Control Conference, 8-11 August 2011, Portland, Oregon (AIAA 2011-6221).

8 W. Green and P. Oh, "A MAV That Flies Like and Airplane and Hovers Like a Helicopter". Proc. 2005 IEEE/ASME International Conference on Advanced Intelligent Mechatronics. 24-28 July Monterey, California.

9 A. Frank, M. Valenti, D. Leveine, J. How. "Hover Transitions and Level Flight Control Design for a Single-Propeller Indoor Airplane", Proc. AIAA Guidance Navigation and Control conference and Exhitbit, August 2007, Hilton Head South Carolina.

${ }^{10}$ E. Johnson and S. Kannan, “ Adaptive Flight Control for an Autonomous Unmanned Helicopter", Proc. AIAA Guidance, Navigation, and Control Conference, 5-8 August 2002, Monterey California . (AIAA 2002-4439)

11 D. Yeo, J. Henderson, and E Atkins, "An Aerodynamic Data System for Small Hovering Fixed-Wing UAS," Proc. AIAA Guidance, Navigation, and Control Conference, 10-13 August 2009, Chicago Illinois. (AIAA 2009-5756)

12 D. Yeo, E. Atkins, L. Bernal and W. Shyy, "Aerodynamic Sensing for a Fixed Wing UAS Operating at High Angles of Attack," Proc. AIAA Atmospheric Flight Mechanics Conference, 13-16 August 2012, Minneapolis Minnesota.(AIAA 2012-4416)

13 M. Patel, Z. Sowle, T. Corke and C. He, "Autonomous Sensing and Control of Wing Stall Using a smart Plasma Slat", Proc. 44 ${ }^{\text {th }}$ AIAA Aerospace Sciences Meeting, 9-12 January 2006, Reno Nevada, (AIAA 2006-1207)

14 P.Bowles and T. Corke. "Stall Detection on a Leading-edge Plasma Actuated Pitching Airfoil Utilizaing Onboard Measurement", Proc. $47^{\text {th }}$ Aerospace Sciences Meeting, 5-8 January 2009, Orlando Florida. (AIAA 2009-93)

15 C. Cox, A. Gopalarathnam, C. Hall. "Flight Test of Stable Automated Cruise Flap for an Adaptive Wing Aircraft". Journal of Aircraft, Vol. 47, No. 4, 2012 pp 1178-1188.

16 W. Barnwell directed by N. Chokani and C. Hall. "UAV Flight Control Using Distributed Actuation and Sensing". Masters Thesis, North Carolina State University 2003.

17 S. Lion directed by C.Hall. "Control Authorities of a distributed Actuation and Sensing Array on a Blended-Wing-Body Uninhabited Aerial Vehicle". Masters Thesis, North Carolina State University 2007.

18 Air Force Office of Scientific Research (AFOSR) Multidisciplinary University Research Initiative (MURI) AFOSR Grant FA9550-05-1-0411, [online] http://www.avocet.gatech.edu 
19 E. Morelli and R. DeLoach, "Wind Tunnel Database Development Using Modern Experiment Design and Multivariate Orthogonal Functions," Proc. 41 ${ }^{\text {st }}$ Aerospace Sciences Meeting and Exhibit, 6-9 January 2003, Reno, Nevada. (AIAA 2003-653)

${ }^{20}$ R. Guiler and W. Huebsch, "Wind Tunnel Analysis of a Morphing Swept Wing Tailess Aircraft," Proc. AIAA Applied Aerodynamics Conference, 6-9 June 2005, Toronto, Ontario, Canada. (AIAA 2005-4981)

${ }^{21}$ P. Marmier and N. Wereley, "Morphing Wings of a Small Scale UAV Using Inflatable Actuators for Sweep Control," Proc. 44 ${ }^{\text {th }}$ AIAA Structures, Structural Dynamics and Materials Conference, 7-10 April 2003, Norfolk, Virginia (AIAA 2003-1802)

22 D. Landman, C. Britcher, and W.Bennet, " A Power-On Full-Scale Wind Tunnel Test of a UAV," Proc. $17^{\text {th }}$ Applied Aerodynamics Conference, Norfolk Virginia (AIAA-99-3166)

${ }^{23}$ M. Ol, C. Zeune, T. White and T. Kudla, "Wind Tunnel Evaluation of Powered Static Aerodynamics of an Aerobatic UAV," Proc. 51 ${ }^{\text {st }}$ Aerospace Sciences Meeting, 7-10 January 2013, Grapevine, Texas ( AIAA 2013-0241)

${ }^{24}$ D. Yeo E. Atkins, L. Bernal and W. Shyy, "Experimental Investigation of the Pressure, Forces and Torque Characteristics of a Rigid Flapping Wing", Proc. 50 $0^{\text {th }}$ AIAA Aerospace Sciences Meeting, January 2012, Nashville TN. (AIAA 2012-0849)

25 N. McClamroch, Steady Aircraft Flight and Performance, Princeton University Press, 2011. 